\title{
Functional Design in Rehabilitation: Modular Mechanisms for Ankle Complex
}

\author{
Francesco Aggogeri, Nicola Pellegrini, and Riccardo Adamini \\ Department of Mechanical and Industrial Engineering, University of Brescia, Via Branze 38, 25123 Brescia, Italy \\ Correspondence should be addressed to Francesco Aggogeri; francesco.aggogeri@unibs.it
}

Received 29 April 2016; Revised 22 June 2016; Accepted 29 June 2016

Academic Editor: Tadeusz Mikołajczyk

Copyright (C) 2016 Francesco Aggogeri et al. This is an open access article distributed under the Creative Commons Attribution License, which permits unrestricted use, distribution, and reproduction in any medium, provided the original work is properly cited.

\begin{abstract}
This paper is aimed at presenting an innovative ankle rehabilitation device based on a parallel mechanism. A functional analysis and design are described to obtain a device able to guarantee ankle movement while patient's body remains stationary. Human ankle is a challenging context where a series of joints are highly integrated. The proposed rehabilitation device permits a patient with walking defects to improve his or her gait. The research focuses on plantar-flexion-dorsiflexion movement. The robust design starts from an accurate modelling of ankle movements during walking, assessing motion data from healthy individuals and patients. The kinematics analysis and functional evaluations lead the study and development of the articulated system. In particular, results of simulations support the effectiveness of the current design. A 3D prototype is presented highlighting that the ankle motion is successfully demonstrated.
\end{abstract}

\section{Introduction}

Rehabilitation engineering is aimed at studying and integrating the human joint movements into equipment optimization in developing effective rehabilitation devices. In this way, the study of human movements [1] plays an important role in creating innovative systems able to satisfy patient requirements in physiotherapy programs. There are many challenges and opportunities to integrate applied mechanics concepts into rehabilitation improving physiotherapy effectiveness and reducing healthcare costs. A new prototype for ankle rehabilitation is presented in this study. It has been designed focusing on the integration of human joint movements with machine architecture for rehabilitation machines. Starting from the patients' requirements and an assessment of ankle motion analysis, this paper introduces the mechanism evaluation and the main technical solutions in the device development. Ankle injuries are one of the most common and serious issues in daily activities and sports (15-20\% of sport injuries) [2]. Therefore, the creation of an apparatus suitable for the ankle physiotherapy requires an awareness of ankle functionalities, in particular the joint motion ones. In any robotic rehabilitation equipment changes in joint motion are taken into account considering the structure motion and the desired increased range-of-ankle motion that can be fulfilled through the use of a device based on parallel apparatus design. A parallel mechanism has the advantages of high dynamic performance supporting the ankle motion. This paper investigates the human ankle complexity, movement, and functional needs in rehabilitation. Ankles injuries are analyzed and physiotherapy protocols are examined. The ankle movement is provided as an orientation where a range is given for ankle rehabilitation. This particular range is used for the concept of equipment based on the angle performance [3]. Further kinematics and stiffness analysis are performed on this innovative system. The research uses interdisciplinary approach, combining the clinical requirements with the parallel mechanism configuration and planning rehabilitation motion in a defined workspace for the aim of concurrent design.

In last decades, many systems have been proposed to recover ankle functionalities. These mechanisms focus on human motions without walking. State of the art presents different approaches in attempting this topic; in particular Girone et al. [4] developed a mechanism based on Stewart platform that permits an assisted ankle rehabilitation using 
double-acting pneumatic cylinders, linear potentiometers, and force sensors. Yano et al. presented a leg-shaped robot of two 2-DOF manipulators equipped with footpads that guarantee movement of feet $[5,6]$. Yoon et al. proposed a reconfigurable ankle rehabilitation robot to cover various rehabilitation exercise modes [7]. In particular, this study shows a device based on a parallel mechanism that generates pitch and roll motions as well as relative rotation between fore and rear platforms. Instead, Dai et al. introduced several parallel mechanisms with a central strut for ankle [8]. In this way, state of the art highlights a broad range of valid solutions; nevertheless there are still many points to be investigated to optimize the trade-off between therapy effectiveness and system automatization.

This paper wants to propose a new device that differs from the other solutions. It is based on a parallel mechanism (1 DOF) able to perform trajectories similar to patient's ankle in nominal environment conditions and permitting to patient's body to remain stationary.

\section{Ankle Injuries Assessment and Movements}

An ankle movement is covered by two separate joints. The first is the articulation among the tibia, the fibula, and the talus, called ankle joint. The second one formed by the talus and the calcaneus is the subtalar articulation [11]. In physiotherapy, the direction, the speed, and the range of joint motion can be altered by injuries to muscles and ligaments and medical treatment relies heavily on an understanding of the normal and abnormal joint functions [12]. The ankle joint is modelled as a simple hinge articulation. During gait cycle, the joint allows inversion and eversion of the foot (passive-resistive function) and it plays an active role in dorsiflexion or plantar-flexion of the foot in nominal environment conditions that represent a significant part of ankle movement. The ankle and subtalar joints have different functionalities and thus their role is critical in developing an excessive ankle ligament stresses.

The ankle joint allows the movements in all 3 body planes (sagittal, frontal, and transverse). The first plane is identified by $x, z$ and rotations around the $y$-axis; the transverse plane is given by $x, y$ and rotations in the $z$-axis; finally the frontal plane is limited by $y$ and $z$. Nowadays, therapies and rehabilitation methods aiming to progress mobility have to work into specific tasks, customized and time optimized. Well-defined injuries have been tested in groups of patients. Improvements are noted in individuals who received a physiotherapy effort within 3 months in comparison to a conventional one that normally requires from 6 up to 8 months [13]. These studies confirm the potential of a target leading physical therapy.

\section{Concurrent Model Driven by Functional Requirements}

Ankle is a sensitive structural and complex system. This study proposes a robust technique able to support rehabilitation therapy in every distinct phases: assessment, training, and control. The starting point is to identify the real-time patient's

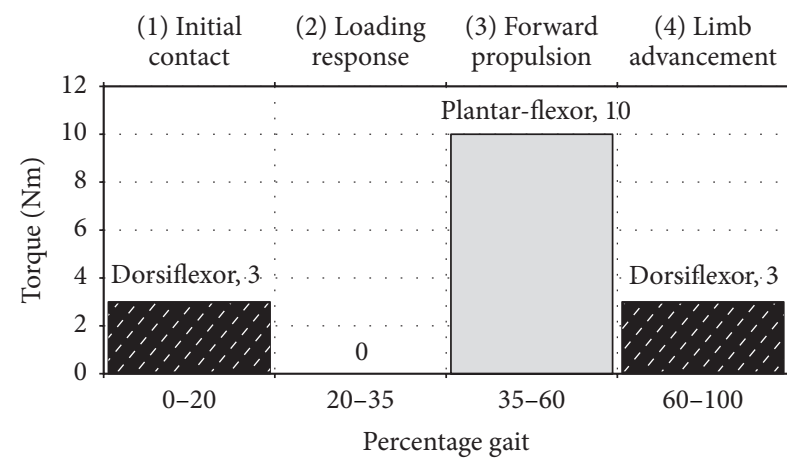

FIGURE 1: Dorsi- and plantar-flexor torque in no-defect walk.

measurements as inputs in generating a specific task. The robot software compares the planned torque or trajectory achieved during rehabilitation. Finally, the robot interface performs the possible correction through the virtual environment. The main assumption is the ability in tracing accurately the joint's center location during the gait cycle.

The developed mechanism provides many types of targeted activities in order to add benefits for autonomous operation, improve safety, manage real-time feedbacks, and reduce the therapist contribution [14]. A preliminary study of gait trajectories using a simple limb model is presented.

The objective of this paper is to develop a rehabilitation device starting from an accurate biomechanical model. Some assumptions of stiffness, stresses, and other limitations are made [15] and included in simulations for the novel iterative roadmap. The framework uses a series of rotations to determine the angle. The $X Y Z$ Euler angles convention is adopted and the orientation of the ankle is described by a rotation about $x$-, $y$-, and $z$-axis.

The plantar-flexion/dorsiflexion operation is expressed by $X$ Euler angle; the inversion/eversion is illustrated by $Y$ Euler one and the abduction/adduction is explained by $Z$ Euler one. The controlled axis is $x$ plantar-flexion/dorsiflexion movement; the other axes are not included. This particular order is chosen considering the clinical applications. Figure 1 indicates the assistive torque at ankle joints by the rehabilitation device in a gait cycle [16]. The needed torque varies from $3 \mathrm{Nm}$ in dorsiflexion operation, while, during impulse phase, the plantar-flexor actuations achieve torques equal to $10 \mathrm{Nm}$ [17].

Nowadays, all commercially rehabilitation robots are composed of joints without any power feedbacks. Passive systems have the benefit to reduce cost guaranteeing the structural simplicity $[18,19]$. These devices are not able to offer assistance during third phase, where additional torque is required. In this case, the motion control is generally based on the actuators in an open loop, when the strength and effectiveness are not guaranteed.

Rehabilitation devices are aimed at recovering people with decreased motion control abilities. This biomechanical model needs additional inputs such as human measures and end effector specifications [20]. The developed iterative 


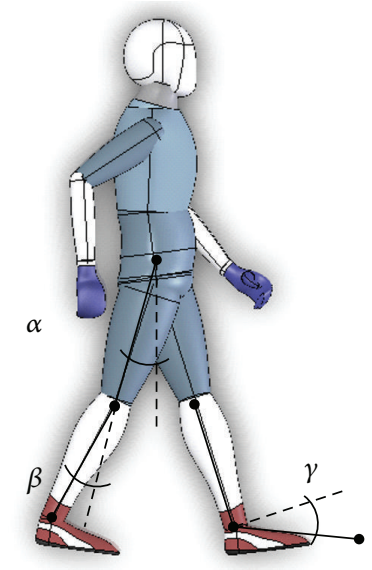

FIGURE 2: Joint angles definition.

approach to define load and trajectory requirements is represented as follows.

(1) Determine patient's parameters with anthropometric indexes.

(2) Estimate joint positions, velocities, accelerations, and loads.

(3) Obtain end effector coordinates from kinematics relationships.

(4) Joint variables are used in the limb's motion equations to acquire the end effector payloads.

Each limb can be described as a junction of three segments representing the foot (from hallux to ankle joint), leg (from ankle to knee), and thigh (from the knee to the hip). Figure 2 highlights anatomical angles of joints flexion in the sagittal plane:

(i) hip flexion angle $(\alpha)$ : the angle between the thigh and the perpendicular line to the ground;

(ii) lower leg bending angle $(\beta)$ : angle between the leg and the line through the thigh;

(iii) foot flexion angle $(\gamma)$ : angle between the line anklehallux and the perpendicular line to leg.

These angles can vary during the gait cycle; in particular they are a function of the gait cycle.

The rehabilitation system workspace needs to be suitable for a wide cluster of people as well as a different range of operation, for instance, walking or stair climbing. Angle and payload limits are used to determine the specified workspace boundary. Table 1 shows the statistical values of these variables [10].

A study on step distance and its relation with morphometric features in adult male is presented. Figure 3 underlines the height for individuals from $1,63 \mathrm{~mm}$ to $1,88 \mathrm{~mm}$ which corresponds to 1st and 99th percentile of male "20 years and over" age with "all race and ethnicity group" of American adults [10]. Other researches considering more than 200 individuals with no walking defects (group age of 20-30 years)
TABLE 1: Range of human ankle motion [10].

\begin{tabular}{lccc}
\hline \multirow{2}{*}{ Movement } & \multicolumn{3}{c}{ Max ankle angles } \\
& Range & Mean & Standard deviation \\
\hline Dorsiflexion & $20.3^{\circ}$ to $29.8^{\circ}$ & $24.6^{\circ}$ & $3.2^{\circ}$ \\
Plantar-flexion & $37.6^{\circ}$ to $45.7^{\circ}$ & $40.9^{\circ}$ & $4.3^{\circ}$ \\
Inversion & $14.5^{\circ}$ to $22.0^{\circ}$ & $16.3^{\circ}$ & $3.8^{\circ}$ \\
Eversion & $10.0^{\circ}$ to $17.0^{\circ}$ & $15.8^{\circ}$ & $4.4^{\circ}$ \\
Internal rotation & $22.0^{\circ}$ to $36.0^{\circ}$ & $29.8^{\circ}$ & $7.5^{\circ}$ \\
External rotation & $15.4^{\circ}$ to $25.9^{\circ}$ & $22.0^{\circ}$ & $5.9^{\circ}$ \\
\hline
\end{tabular}

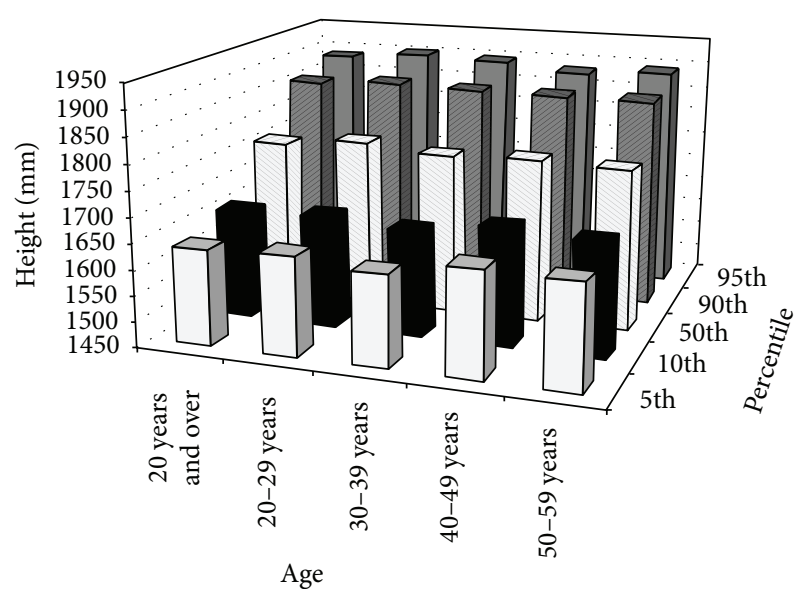

FIGURE 3: Height in millimeters for males by age and selected percentiles.

note the step distance between $600 \mathrm{~mm}$ and $700 \mathrm{~mm}$ range interval [21].

The main result of this analysis is the correlation between the step distance and the stature, investigated in many studies [22]. A set of methods are usually applied to capture accurate motion data from healthy individuals and patients [2325]. In particular optical measurement systems, force plates, wearable sensors, accelerometers, and gyroscopes are applied to measure gait parameters, as shown in different researches $[26,27]$. In this way, the user's needs are considered in designing and developing the rehabilitation devices [28]. It is possible to recap the ankle model coordinates to the step length (hereafter as $L_{p}$ ). In order to study the ankle kinematics it is necessary to compare the $x$-and $y$-axis to the step length of the patient obtaining, respectively, $A_{i}$ and $B_{i}$ index:

$$
\begin{aligned}
A_{i} & =\frac{X_{i} \text { ankle }}{L_{p}} ; \\
B_{i} & =\frac{Y_{i} \text { ankle }}{L_{p}} .
\end{aligned}
$$

In the following equation the kinematics of any patient of known stature is determined as follows:

$$
\begin{gathered}
X_{i} \text { ankle }=A_{i} \cdot L_{p} ; \\
Y_{i} \text { ankle }=B_{i} \cdot L_{p} .
\end{gathered}
$$




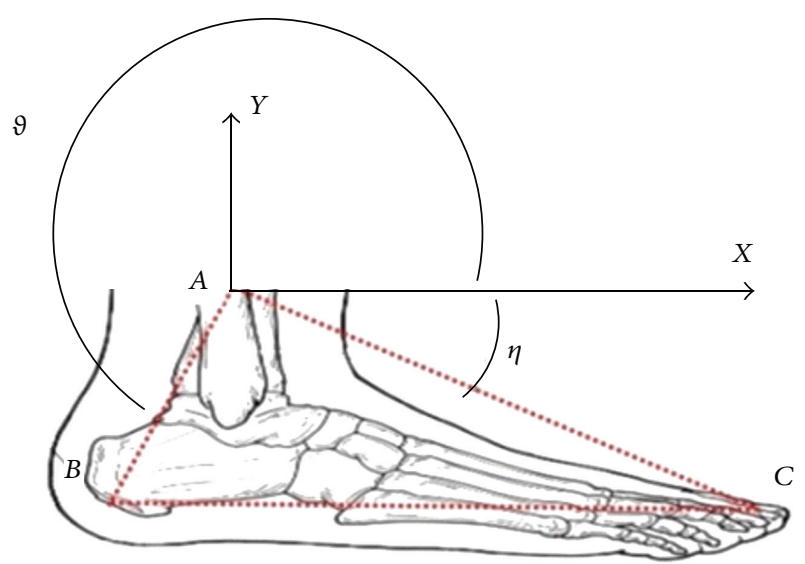

FIGURE 4: Kinematic model angles: $\eta$ from the ground parallel to hallux and $\theta$ from ground parallel to heel [9].

In order to define this trajectory it is necessary to assume the foot as a triangle with vertices at the hallux, ankle, and heel (Figure 4). Then it is needed to define the two angles:

(i) $\eta$ : the angle from the ground parallel to the ankle and hallux,

(ii) $\theta$ : the angle from the ground parallel to the ankle and heel.

It is possible to estimate the angle by considered instance of time, knowing the coordinates of the vertices of the foot by

$$
\begin{gathered}
\eta=\arctan \frac{Y_{i} \text { hallux }-Y_{i} \text { ankle }}{X_{i} \text { hallux }-Y_{i} \text { ankle }} \\
\vartheta=\pi+\arctan \frac{Y_{i} \text { heel }-Y_{i} \text { ankle }}{X_{i} \text { heel }-Y_{i} \text { ankle }} .
\end{gathered}
$$

The assumption for walk trajectory planning is based on $\eta$ and $\vartheta$ angles as independent of patients and step distance. In fact, it is sufficient to know the distance of ankle-hallux $(C A)$ and ankle-heel $(A B)$ of a patient in order to determine the standard kinematics of the step:

$$
\begin{gathered}
\operatorname{Hallux}(X, Y)=[X \text { ankle }+C A \cdot \cos (\eta) ; Y \text { ankle }+C A \cdot \sin (\eta)] ; \\
\operatorname{Heel}(X, Y)=[X \text { ankle }+A B \cdot \cos (\eta) ; Y \text { ankle }+A B \cdot \sin (\eta)] .
\end{gathered}
$$

The model obtained represents the typical walk of a healthy subject. This is the starting point for a preliminary trajectory definition that will be customized in order to identify ad hoc tasks and training.

In this paper the purpose is to analyze the kinematics of the ankle on the plantar-flexion movement and to increase the degree of patients' mobility. A cyclic trajectory comparing the kinematics of the ankle needs to be defined with a particular articulated mechanism, referring to a person with no walking defects.

The oscillatory phase of the gait cycle is the ankle kinematics, while the support phase is replaced and approximated by a constant motion of the ankle through the initial position

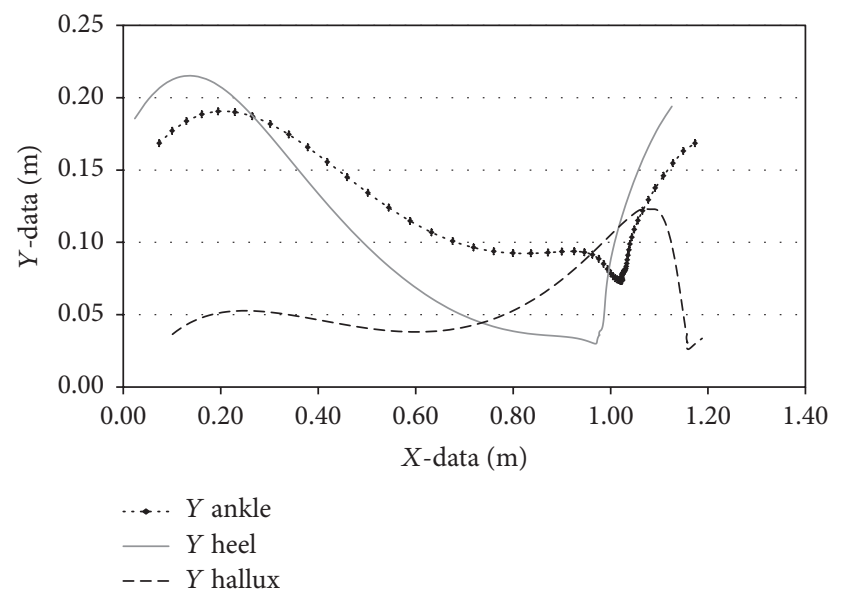

FIGURE 5: Spatial trajectory in transverse plane during gait cycle.

of the cycle. Figures 5 and 6 describe the trajectory and the velocity of the ankle, heel, and hallux model considered during the step cycle in the related Cartesian plan.

\section{Modular Mechanism Concept and Simulation}

The know-how of the mechanism kinematics and loading performance are necessary inputs to identify the architecture. An iterative process is proposed in order to find the most advantageous mechanism design configuration.

The aim of the paper is to obtain a smart architecture of the mechanism (1 degree of freedom) able to perform trajectories similar to patient's ankle. The software used for the simulation of the planar apparatus is MECAD sw. This configuration allows the usage of one motor performing the movement of the articulated system. The mechanical equipment is constituted by 2 rods and 3 constraints; see Figure 7. Degrees of freedom for planar linkages joined with common joints can be calculated through Gruebler's equation [29]:

$$
\mathrm{DOF}=3 \cdot(n-1)-2 \cdot j_{p}-j_{h}=6-5=1 \text {, }
$$

where $n$ is the overall sum of links in the mechanism, $j_{p}$ is the total number of primary joints, and $j_{h}$ is the amount of higher-order joints.

Figure 8 shows an overview of the articulated mechanism where link $5-6$ is the crank shaft while link 3-5 is the piston rod. The main system parameters considered in the final simulation are listed in Table 2. A set of assumptions and constraints has been identified in defining the device design and parameters. For example, the rehabilitation walking step cannot exceed $555 \mathrm{~mm}$ in order to guarantee patient's safety and therapy effectiveness, as extrapolated from gait analysis.

The use of MECAD permits comparing different configurations with perturbed parameters [30-33]. The simulation output is shown in Figure 9. The proposed physical prototype of a novel modular ankle rehabilitation system is presented in 
TABLE 2: Parameters of the articulated system.

\begin{tabular}{|c|c|c|c|c|c|c|c|}
\hline Walking step [mm] & Link 5-6 [mm] & Link 3-5 [mm] & Distance 3-7 [mm] & $\lambda$ & $\varphi$ & $\zeta$ & $\varepsilon$ \\
\hline 554.80 & 191.40 & 390.30 & 155.90 & $65.82^{\circ}$ & $9.86^{\circ}$ & $96.12^{\circ}$ & $7.52^{\circ}$ \\
\hline
\end{tabular}

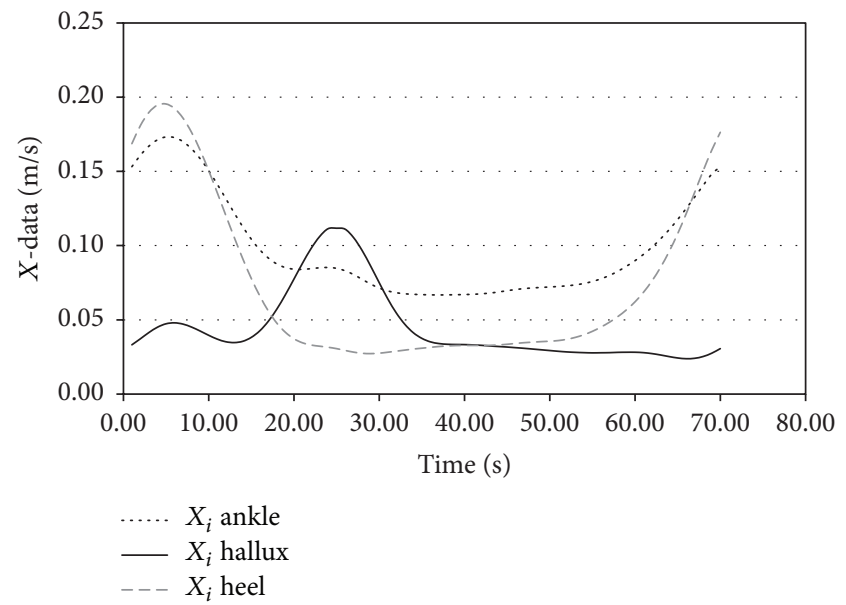

(a)

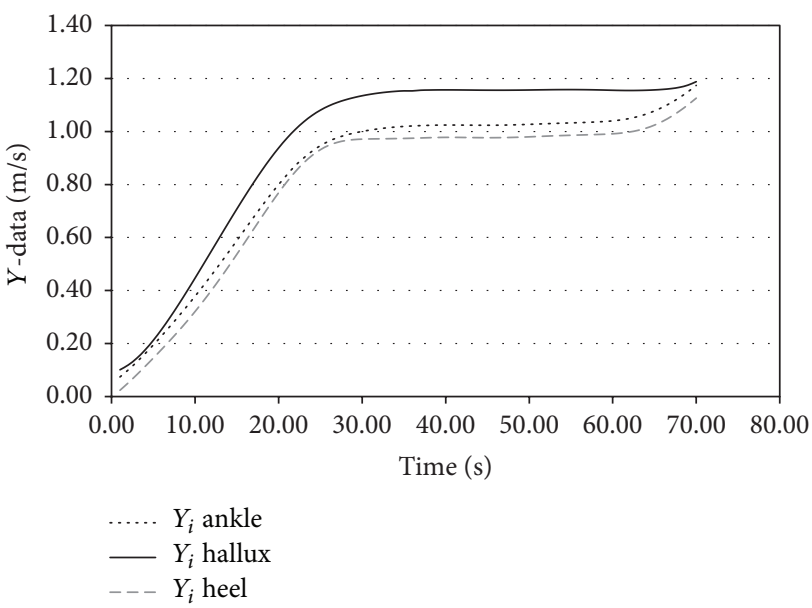

(b)

Figure 6: Trajectory in $X$-plane (a) and $Y$-plane (b) during rehabilitation task.
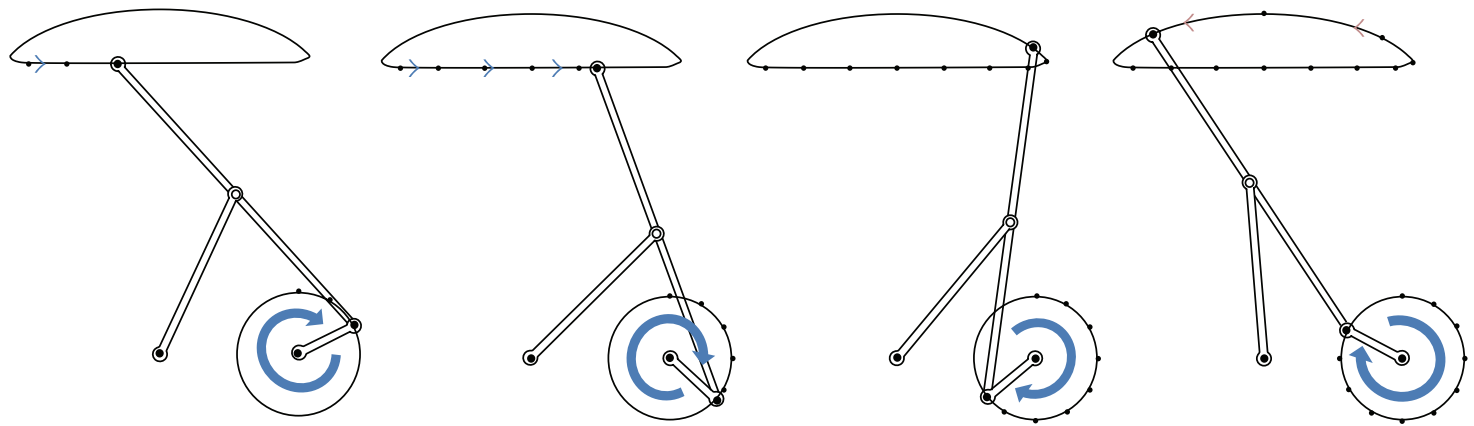

FIGURE 7: Noneccentric crank with single degree of freedom.

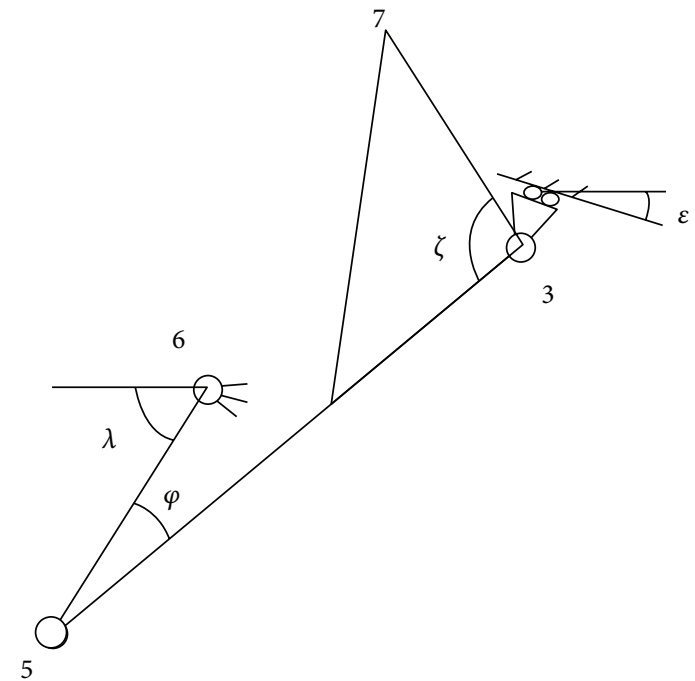

FIGURE 8: An overview of the articulated mechanism.
Figure 10. The design is composed of one motor that transmits power to both left and right limb.

The main benefits of the proposed rehabilitation system are as follows:

(1) Modular design: individual customization is the adding-value due to trajectory/kinematics parameters setting and actuation system selection. For instance, the baseline is the walking cycle time of a healthy person close to 2 seconds while the duration for a patient can greatly vary depending on several factors (accident severity, rehabilitation stage, age, etc.).

(2) Reliability and control: the system architecture based on a parallel mechanic can guarantee precision of movement while the patient's body remains stationary. In particular, this configuration permits controlling and monitoring the device easily (e.g., using 


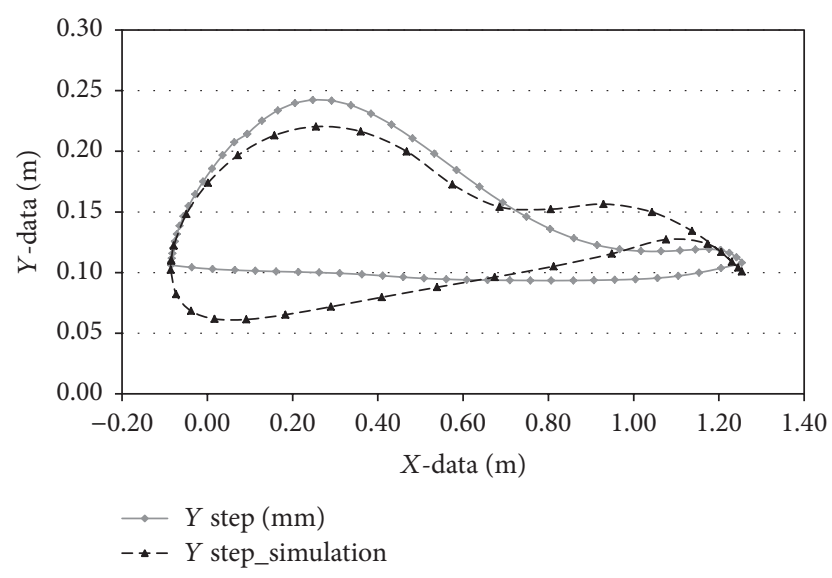

FIgURE 9: Comparison between measured and simulated trajectory in transverse plane during gait cycle.

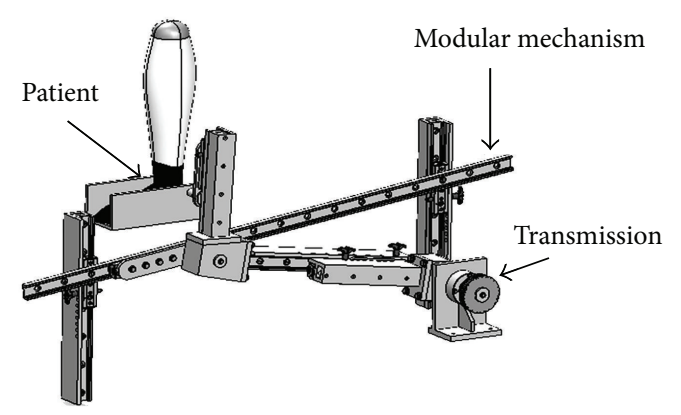

FIgURE 10: Modular mechanism system for ankle complex.

feedback signal strategy) providing an effective and robust therapy.

(3) Repeatability of movement: the device guarantees the repeatability of movement in particular for aged people.

(4) Adaptive control: usually the rehabilitation robots are position-based control, while this innovative control method is structure-based to provide torque assistance. The presented controller covers online learning platform able to monitor individual user performances during different rehabilitation gates.

(5) Errorless Learning approach: this device allows the creation of movement trajectories that encourage "Errorless Learning" approach where the patient needs to take an active part in movement initiation and execution. In fact it is often difficult to teach patient the correct motion of leg, specialty for aged people.

(6) Autonomous operation reducing the therapist contribution: patient can perform the rehabilitation exercise independently and easily, reducing the therapist's effort and guaranteeing the effectiveness of rehabilitation and safety.
(7) Light-weight of structure and compactness design with an impact on commercialization and usability of device in medical applications.

In the light of these preliminary results, the next steps of the research will be based on further improvements of the rehabilitation device in terms of functionality and patients' safety. In particular a failure mode and effect analysis is suggested to identify all potential criticalities of system. This evaluation needs to consider and avoid any issues that may impact patients' safety during rehabilitation therapy (e.g., unexpected error of the system). The system will be equipped with footpads, additional lanes to relieve limbs, and a safety bar placed around the system to assure patient's safety during the rehabilitation exercises. The design review will identify the suitable actuation and control strategy. A preliminary evaluation suggested to use a feedback signal strategy in order to improve the therapy cycle controlling the main system parameters. This strategy permits customizing the therapy and guaranteeing the required movement. In particular, as shown in functional analysis, the walking cycle time (CT) of a healthy adult is close to $2 \mathrm{sec} / \mathrm{step}$, while it may vary for a patient based on therapy. Assuming a very slow therapy $(\mathrm{CT}=4 \mathrm{sec})$ and the worst case in terms of load (patient's weight equal to $150 \mathrm{~kg}$ ), it is possible to simulate and calculate the maximum torque for system actuation. A preliminary calculation estimated a required torque close to $206 \mathrm{Nm}$ considering whole mechanism and a safety factor equal to 1.2. The results of this further investigation will provide the system configuration suitable for prototype realization. The final purpose is to test and validate the rehabilitation device involving patients, physiotherapists, and physicians.

\section{Conclusion}

This paper introduces an ankle kinematic model and it uses this knowledge to design a device based on a parallel mechanism. This investigation focuses on the integration of human joint movements into rehabilitation device development in order to increase performance and effectiveness. The validity on physiotherapy of gait has been studied, modelling ankle movements during walking and assessing motion data from healthy individuals and patients. Using a functional analysis a preliminary rehabilitation system is proposed able to provide and guarantee the rehabilitation cycle and torque assistance during the required therapy. The study describes the technical features and requirements of the proposed device to guarantee patient's safety and therapy effectiveness. Using software simulations the ankle motion is successfully demonstrated by the rehabilitation system. Further investigations are required to complete and optimize the device, avoiding any potential issue that may impact patients' safety during rehabilitation exercise. In particular, a specific study will be performed to identify the most suitable footpads to guarantee an effective movement and a safe therapy. The design review will also suggest the correct actuation and control strategy. A preliminary study highlighted that the feedback control is a suitable method to improve the system performances. The final step is to propose a prototype to be 
tested involving patients, physiotherapists, and physicians. Experimental validations on patients or healthy individuals play a key role in the effective application of the rehabilitation systems. The possibility to include analysis performed on patients should improve the dynamic control and the clinical effectiveness quantifying the ability and the progress during training process. A set of limits and constrains are still under investigation; in particular future works need also to focus on more complex and realistic environment training.

\section{Competing Interests}

The authors declare that they have no competing interests.

\section{References}

[1] H. Kawamoto and Y. Sankai, "Comfortable, power assist control method for walking aid by HAL-3," in Proceedings of the IEEE International Conference on Robotics and Automation, vol. 4, October 2002.

[2] M. Nordin and V. H. Frankel, Basic Biomechanics of the Musculoskeletal System, Lippincott Williams \& Wilkins, Philadelphia, $\mathrm{Pa}, \mathrm{USA}$, 3rd edition, 2001.

[3] D. Simsik, M. Drutarovsky, P. Galajda, and A. Galajdova, "Embedded control of mechatronical rehabilitation shoe," Engineering. Media/ST. Zilina, vol. 15, no. 5, pp. 1-6, 2011.

[4] M. Girone, G. Burdea, M. Bouzit, V. Popescu, and J. E. Deutsch, "Stewart platform-based system for ankle telerehabilitation," Autonomous Robots, vol. 10, no. 2, pp. 203-212, 2001.

[5] H. Yano, S. Tamefusa, N. Tanaka, H. Saitou, and H. Iwata, "Gait rehabilitation system for stair climbing and descending," in Proceedings of the IEEE Haptics Symposium (HAPTICS '10), pp. 393-400, IEEE, Waltham, Mass, USA, March 2010.

[6] T. Kikuchi, K. Oda, and J. Furusho, "Leg-robot for demonstration of spastic movements of brain-injured patients with compact magnetorheological fluid clutch," Advanced Robotics, vol. 24, no. 5-6, pp. 671-686, 2010.

[7] J. Yoon and J. Ryu, "A novel reconfigurable ankle/foot rehabilitation robot," in Proceedings of the IEEE International Conference on Robotics and Automation (ICRA '05), pp. 2290-2295, IEEE, Barcelona, Spain, April 2005.

[8] J. S. Dai, T. Zhao, and C. Nester, "Sprained ankle physiotherapy based mechanism synthesis and stiffness analysis of a robotic rehabilitation device," Autonomous Robots, vol. 16, no. 2, pp. 207-218, 2004.

[9] R. P. Wells, "The projection of the ground reaction force as a predictor of internal joint moments," Bulletin of Prosthetics Research, vol. 18, no. 1, pp. 15-19, 1981.

[10] A. H. Hansen, D. S. Childress, S. C. Miff, S. A. Gard, and K. P. Mesplay, "The human ankle during walking: implications for design of biomimetic ankle prostheses," Journal of Biomechanics, vol. 37, no. 10, pp. 1467-1474, 2004.

[11] S. Hesse, H. Schmidt, C. Werner, and A. Bardeleben, "Upper and lower extremity robotic devices for rehabilitation and for studying motor control," Current Opinion in Neurology, vol. 16, no. 6, pp. 705-710, 2003.

[12] F. Sup, A. Bohara, and M. Goldfarb, "Design and control of a powered knee and ankle prosthesis," in Proceedings of the IEEE International Conference on Robotics and Automation (ICRA '07), pp. 4134-4139, Roma, Italy, April 2007.
[13] J. Bae, K. Haninger, D. Wai, X. Garcia, and M. Tomizuka, "A network-based monitoring system for rehabilitation," in Proceedings of the IEEE/ASME International Conference on Advanced Intelligent Mechatronics (AIM '12), pp. 232-237, IEEE, Kachsiung, Taiwan, July 2012.

[14] F. Aggogeri, A. Borboni, and R. Faglia, "Reliability roadmap for mechatronic systems," Applied Mechanics and Materials, vol. 373-375, pp. 130-133, 2013.

[15] M. G. Pandy, "Simple and complex models for studying muscle function in walking," Philosophical Transactions of the Royal Society B: Biological Sciences, vol. 358, no. 1437, pp. 1501-1509, 2003.

[16] D. Bradley, C. Acosta-Marquez, M. Hawley, S. Brownsell, P. Enderby, and S. Mawson, "NeXOS-the design, development and evaluation of a rehabilitation system for the lower limbs," Mechatronics, vol. 19, no. 2, pp. 247-257, 2009.

[17] Y. Hurmuzlu and G. D. Moskowitz, "Bipedal locomotion stabilized by impact and switching," Dynamics and Stability of Systems. An International Journal, vol. 2, no. 2, pp. 73-96, 1987.

[18] J. L. Emken, J. H. Wynne, S. J. Harkema, and D. J. Reinkensmeyer, "A robotic device for manipulating human stepping," IEEE Transactions on Robotics, vol. 22, no. 1, pp. 185-189, 2006.

[19] S. K. Banala, S. H. Kim, S. K. Agrawal, and J. P. Scholz, "Robot assisted gait training with active leg exoskeleton (ALEX)," IEEE Transactions on Neural Systems and Rehabilitation Engineering, vol. 17, no. 1, pp. 2-8, 2009.

[20] A. Agrawal, S. K. Banala, S. K. Agrawal, and S. A. BinderMacleod, "Design of a two degree-of-freedom ankle-foot orthosis for robotic rehabilitation," in Proceedings of the 9th International Conference on Rehabilitation Robotics (ICORR '05), pp. 41-44, IEEE, July 2005.

[21] M. Zinn, O. Khatib, and B. Roth, "A new actuation approach for human friendly robot design," in Proceedings of the IEEE International Conference on Robotics and Automation, vol. 1, pp. 249-254, May 2004.

[22] A. Takanishi, Y. Ogura, and K. Itoh, "Some issues in humanoid robot design," in Proceedings of the International Symposium of Robotics Research, 2005.

[23] H. Saito, T. Watanabe, and A. Arifin, "Ankle and knee joint angle measurements during gait with wearable sensor system for rehabilitation," in Proceedings of the World Congress on Medical Physics and Biomedical Engineering, pp. 506-509, Munich, Germany, September 2009.

[24] J. R. W. Morris, "Accelerometry-a technique for the measurement of human body movements," Journal of Biomechanics, vol. 6, pp. 729-736, 1973.

[25] S. Tadano, R. Takeda, and H. Miyagawa, "Three dimensional gait analysis using wearable acceleration and gyro sensors based on quaternion calculations," Sensors, vol. 13, no. 7, pp. 93219343, 2013.

[26] K. Tong and M. H. Granat, "A practical gait analysis system using gyroscopes," Medical Engineering and Physics, vol. 21, no. 2, pp. 87-94, 1999.

[27] A. M. Sabatini, "Quaternion based attitude estimation algorithm applied to signals from body-mounted gyroscopes," Electronics Letters, vol. 40, no. 10, pp. 584-586, 2004.

[28] E. Gentili, F. Aggogeri, and M. Mazzola, "The effectiveness of the quality function deployment in managing manufacturing and transactional processes," in Proceedings of the ASME International Mechanical Engineering Congress and Exposition (IMECE '07), pp. 237-246, Seattle, Wash, USA, November 2007. 
[29] J. Yoon and J. Ryu, "A novel reconfigurable ankle/foot rehabilitation robot," in Proceedings of the IEEE International Conference on Robotics and Automation, pp. 2290-2295, IEEE, Barcelona, Spain, April 2005.

[30] A. Hreljac and R. N. Marshall, "Algorithms to determine event timing during normal walking using kinematic data," Journal of Biomechanics, vol. 33, no. 6, pp. 783-786, 2000.

[31] H. J. Woltring, "A Fortran package for generalized, crossvalidatory spline smoothing and differentiation," Advances in Engineering Software, vol. 8, no. 2, pp. 104-113, 1986.

[32] F. C. Anderson and M. G. Pandy, "Individual muscle contributions to support in normal walking," Gait and Posture, vol. 17, no. 2, pp. 159-169, 2003.

[33] R. B. Davis, S. Ounpuu, D. Tyburski, and J. R. Gage, "A gait analysis data collection and reduction technique," Human Movement Science, vol. 10, no. 5, pp. 575-587, 1991. 


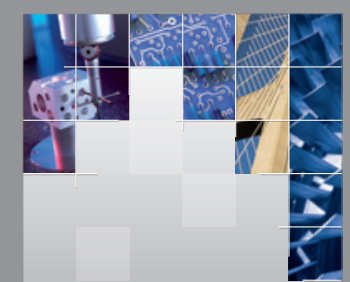

\section{Enfincering}
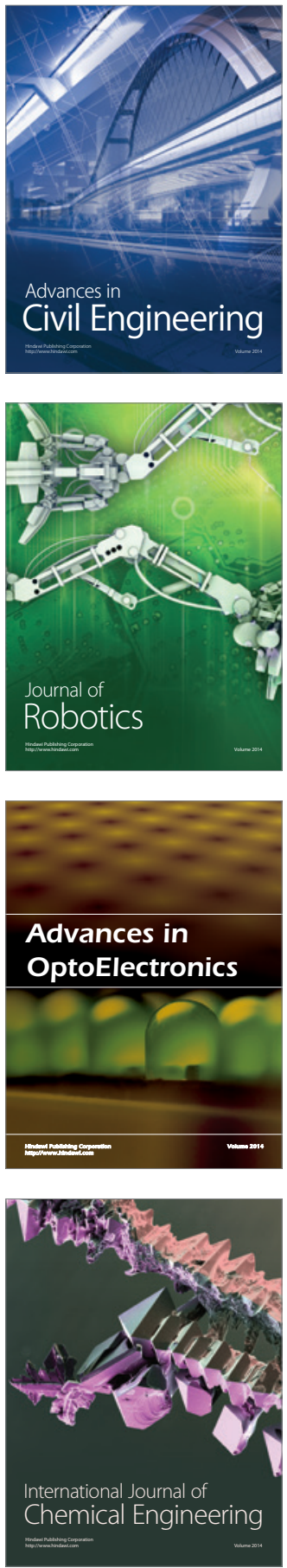

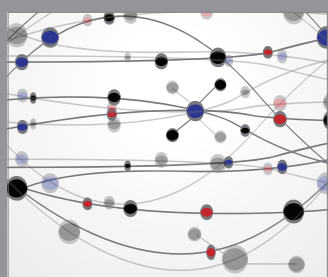

The Scientific World Journal

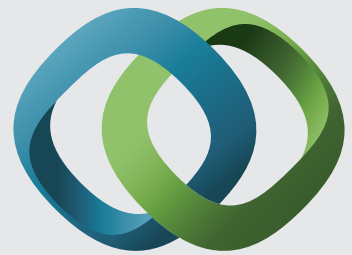

\section{Hindawi}

Submit your manuscripts at

http://www.hindawi.com
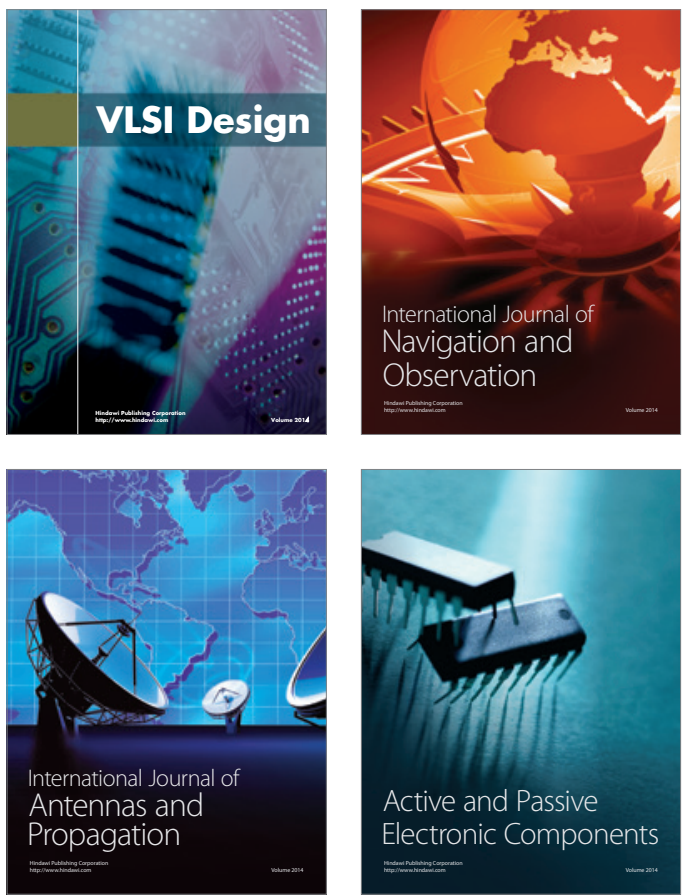
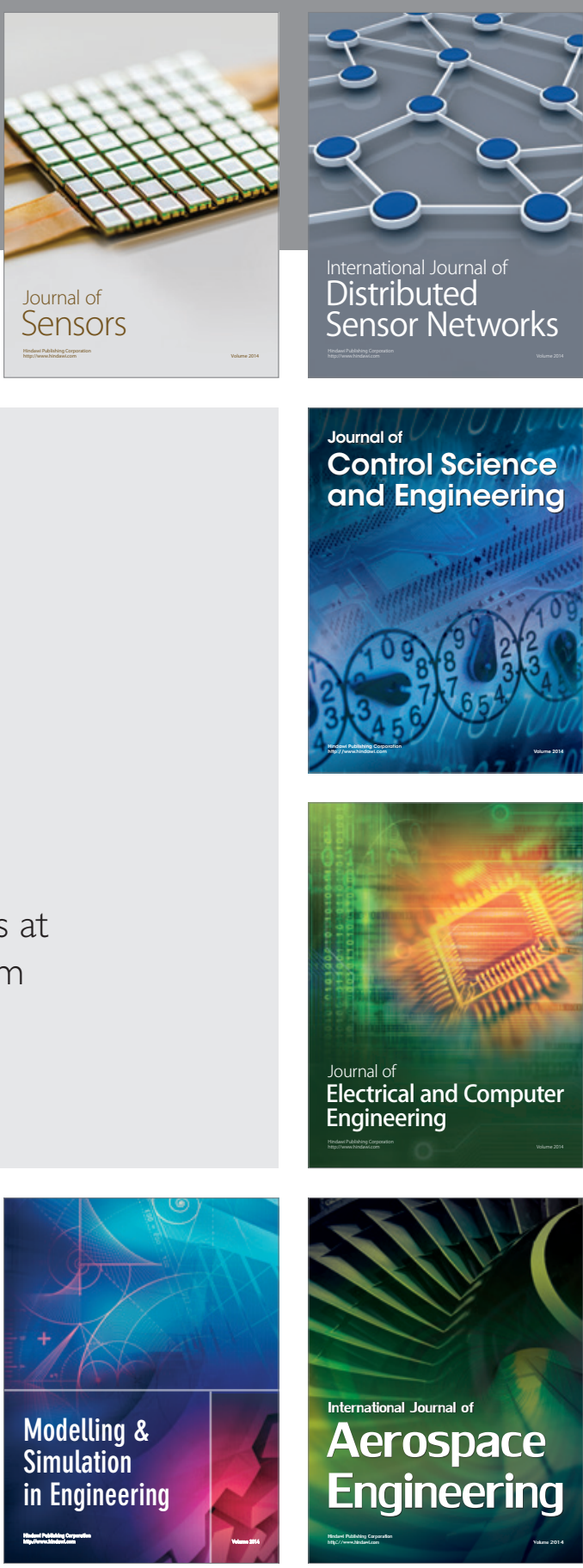

International Journal of

Distributed

Sensor Networks

Journal of

Control Science

and Engineering
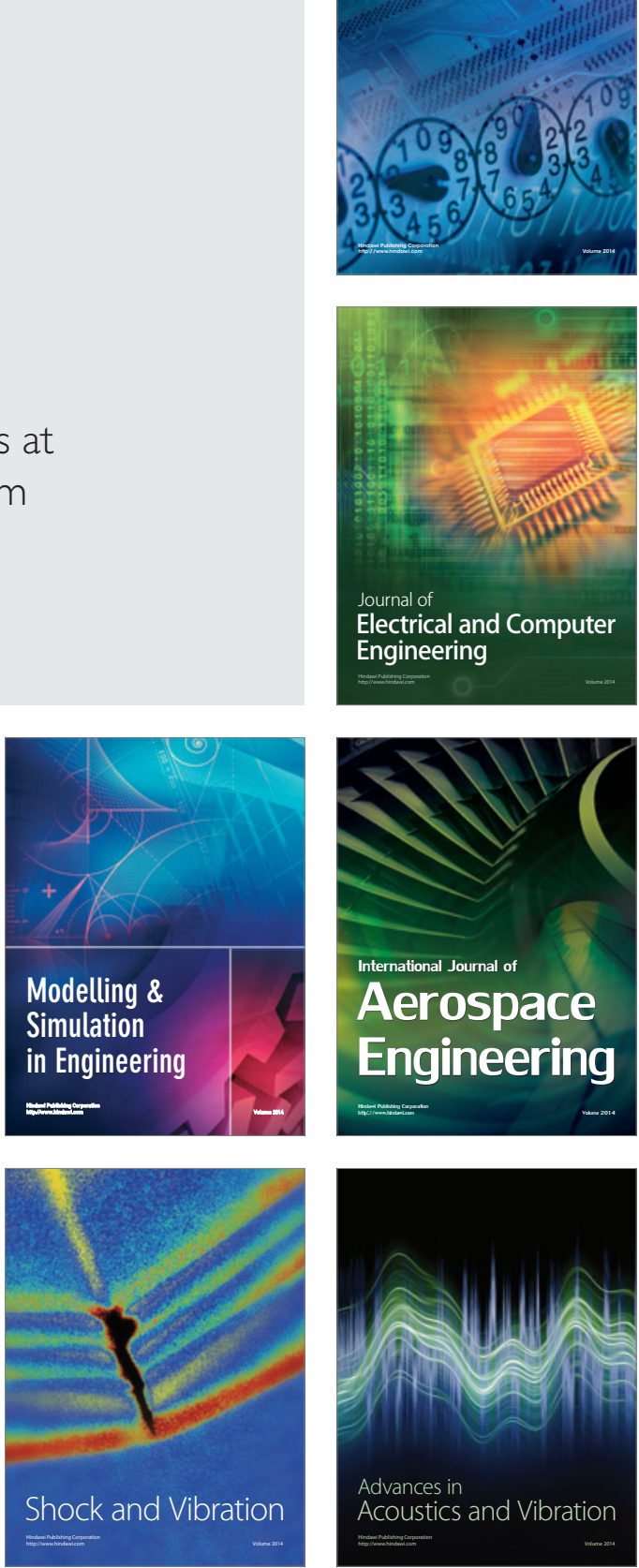